\title{
Proximal humerus reconstruction after tumour resection: biological versus endoprosthetic reconstruction
}

\author{
Michiel A. J. van de Sande • P. D. Sander Dijkstra • \\ Antonie H. M. Taminiau
}

Received: 14 September 2010 /Revised: 19 October 2010 /Accepted: 25 October 2010/Published online: 18 November 2010

(C) The Author(s) 2010. This article is published with open access at Springerlink.com

\begin{abstract}
The purpose of this study was to compare the outcome, complications and survival of the three most commonly used surgical reconstructions of the proximal humerus after transarticular tumour resection. Between 1985 and 2005, 38 consecutive proximal humeral reconstructions using allograft-prosthesis composite $(n=10)$, osteoarticular allograft $(n=13)$ or a modular tumour prosthesis $(n=14)$ were performed in our clinic. The mean follow-up was ten years (1-25). Of these, 27 were disease free at latest follow-up (mean 16.8 years) and ten had died of disease. The endoprosthetic group presented the smallest complication rate of $21 \%(n=1)$, compared to $40 \%(n=4)$ in the allograftprosthesis composite and $62 \%(n=8)$ in the osteoarticular allograft group. Only one revision was performed in the endoprosthetic group, in a case of shoulder instability. Infection after revision $(n=3)$, pseudoarthrosis $(n=2)$, fracture of the allograft $(n=3)$ and shoulder instability $(n=4)$ were the major complications of allograft use in general. Kaplan-Meier analysis showed a significantly better implant survival for the endoprosthetic group (log-rank $p=0.002)$. At final follow-up the Musculoskeletal Tumour Society scores were an average of $72 \%$ for the allograft-prosthetic composite ( $n=7$, median follow-up 17 years), $76 \%$ for the osteoarticular allograft $(n=3,19$ years $)$ and $77 \%$ for the endoprosthetic reconstruction $(n=10,5$ years) groups. An endoprosthetic reconstruction after transarticular proximal humeral resection resulted in the lowest complication rate, highest implant survival and comparable functional results when compared to allograft-prosthesis composite and osteo-
\end{abstract}

M. A. J. van de Sande $(\triangle) \cdot$ P. D. S. Dijkstra • A. H. M. Taminiau Department of Orthopaedics, Leiden University Medical Center, Albinusdreef 2,

2300 RC, Leiden, The Netherlands

e-mail: majvandesande@lumc.nl articular allograft use. We believe that the surgical approach that best preserves the abductor mechanism and provides sufficient surgical exposure for tumour resection contributed to better functional results and glenohumeral stability in the endoprosthetic group.

\section{Introduction}

Limb salvage following tumour resection about the proximal humerus in the adult patient poses significant surgical challenges as it is often limited by surgical loss of surrounding functional soft tissue stabilisers of the glenohumeral joint. Although studies have emphasised the possibility of deltoid muscle and axillary nerve sparing without compromising oncological surgical margins, transarticular tumour resections, including part of the deltoid muscle and rotator cuff, are frequently reported [1-4]. In the adult patient functional reconstruction after transarticular proximal humeral resection is most frequently performed using either osteoarticular allograft (OA), endoprosthesis (EP) or a combination of both [4-6]. As a broad range of functional results and complication rates are reported for all three, the choice for one or the other remains one of surgical experience, time set and local preference [2, 4, 6-8]. Mid- and long-term complications, such as fracture, subchondral collapse and infection, are however reported less frequently in prosthetic reconstructions. Conversely, glenohumeral instability is considered to be less frequent in patients with a somewhat more biological repair. However, in both OA and allograftprosthesis composite (APC) reconstruction, instability caused by rotator cuff dysfunction is reported to be between $5 \%$ and $19 \%$ of cases compared to between $11 \%$ and $31 \%$ after endoprosthetic reconstruction (EPR) [4, 7, 9]. Again, an intact abductor mechanism was associated with increased 
glenohumeral stability. Historical cohort studies comparing different surgical options both concluded that APC is favoured above EPR in young patients, as functional results are somewhat better, but in the older patient EP is preferred for its superior implant survival $[4,6,7]$.

This retrospective cohort series compares three surgical options (EP, OA and APC) for implant- and resectionrelated outcome parameters to determine which type of reconstruction, after primary transarticular resection, could in the long term lead to optimal functional results, patient and implant survival. Additionally, we hypothesised that optimal functional results can be achieved when the deltoid muscle, including the axillary nerve, is left unharmed using a new deltoid-preserving surgical approach.

\section{Patients and methods}

Between 1985 and 2007, 37 consecutive proximal humeral reconstructions using either APC $(n=10), \mathrm{OA}(n=13)$ or a modular tumour prosthesis $(n=14)$ were performed in our clinic. A total of 33 patients underwent limb salvage resection for a primary bone tumour ( 9 benign and 24 malignant) and four for metastatic disease. The histological diagnosis was osteosarcoma in ten, Ewing's sarcoma in two, high-grade chondrosarcoma in 11, giant cell tumour in eight, and malignant fibrous histiocytoma (MFH) and aneurysmatic bone cyst in one. Patients with metastatic disease were treated with EP in three cases and APC in one. Primary bone lesions as described above were evenly divided between all three groups.

All patients were treated with the appropriate (neo) adjuvant chemo- and/or radiation therapy using the appropriate treatment protocols. Chondrosarcomas and benign bone lesions were treated by surgical resection only.

There were 21 male and 16 female patients. Their mean age was 44.8 years (range 16-83).

The mean follow-up was ten years, ranging from nine months to 25 years. None of the surviving patients $(n=28)$ were lost to follow-up (mean follow-up 16.9 years, ranging from 32 months to 25 years).

We retrospectively analysed all medical records for patient characteristics, age at diagnosis, diagnosis, surgical treatment and approach, duration of follow-up, integrity of abductor mechanism, humeral resection length measured from the tip of the greater tuberosity, cortical destruction, resection margins, adjuvant treatment, postoperative complications, oncological parameters including overall survival, and local or systemic relapse. Event-free and implant survival are described using a Kaplan-Meier analysis. Functional assessment at the time of final follow-up was assessed using the Musculoskeletal Tumour Society (MSTS) functional scores for patients still in follow-up (the MSTS score range between 0 and 30 is presented here as a percentage: raw score/30) [10]. The shoulder abductor mechanism was considered compromised when the rotator cuff or axillary nerve was sacrificed and/or greater than $50 \%$ of the deltoid muscle was resected [7].

Patients treated with allograft (OA and APC) were on average ten years younger when compared to the EP group (33, 34 and 44 years, respectively, $p=0.005$ ). Further patient characteristics are summarised in Table 1. Nine patients died of disease (DOD) at a mean of 4.5 years postoperatively (range 2-6 years), and one patient died of hepatocellular carcinoma five years postoperatively.

\section{Surgical technique}

All patients were surgically treated by a single experienced musculoskeletal oncologist (AHMT). The surgical approach was an extended deltopectoral in 13 or a distal deltoid flap approach in 24 shoulders. In contrast to the classic deltopectoral approach, this deltoid flap approach releases the deltoid muscle as one flap, including the overlying skin, from its insertion on the humeral diaphysis to provide for adequate and safe exposure of the complete proximal humerus, axillary and if necessary radial nerve and axillary fossa [11-13]. Using this approach we could spare the deltoid muscle and axillary nerve without sacrificing intended wide surgical margins in all but three patients, in which the axillary nerve was involved in the tumour. All were transarticular resections, leaving the glenoid intact (Table 2). The humeral diaphysis was isolated at least $2 \mathrm{~cm}$ from the most distal part of the lesion and cut using an oscillating saw. If possible the deltoid insertion was, if not already released, spared for later reinsertion. If appropriate the deltoid insertion was released, the axillary nerve was spared and the rotator cuff was cut at its insertion on the native humeral head in order to provide sufficient tendon length for subsequent reconstruction. Reconstructions were performed using techniques described in earlier reports $[6,7]$.

OAs and APCs were templated and ordered preoperatively from a well-established tissue bank (National Bone Bank/BIS Foundation, Leiden, The Netherlands). After aseptic recovery and processing, all allografts for OA and APC reconstructions were fresh-frozen at $-80^{\circ} \mathrm{C}$. To assure optimal articular cartilage integrity none were secondarily sterilised with chemicals or radiation. All EPRs were performed using the MUTARS modular proximal humeral prosthesis (Implantcast, Buxtehude, Germany) and APC reconstructions using a biomodular shoulder prosthesis (Biomet, Warsaw, IN, USA). EPRs were only cemented $(n=4)$ when press-fit fixation was prevented by large diaphysis diameter, using a thirdgeneration technique. APC reconstructions were cemented into the allograft and subsequently fixated to host distal 
Table 1 Surgical and oncological characteristics

\begin{tabular}{|c|c|c|c|c|c|c|c|c|c|c|}
\hline & \multicolumn{2}{|l|}{ Approach } & \multirow{2}{*}{$\begin{array}{l}\text { Axillary } \\
\text { nerve } \\
\text { intact }\end{array}$} & \multirow{2}{*}{$\begin{array}{l}\text { Rotator } \\
\text { cuff } \\
\text { intact }\end{array}$} & \multirow{2}{*}{$\begin{array}{l}\text { Abductor } \\
\text { mechanism } \\
\text { intact }\end{array}$} & \multirow{2}{*}{$\begin{array}{l}\text { Free } \\
\text { (marginal) } \\
\text { margins }\end{array}$} & \multirow{2}{*}{$\begin{array}{l}\text { Mean } \\
\text { resection } \\
\text { (min.-max.) }\end{array}$} & \multirow{2}{*}{$\begin{array}{l}\text { Adjuvant } \\
\text { radiation } \\
\text { therapy }^{\mathrm{a}}\end{array}$} & \multirow{2}{*}{$\begin{array}{l}\text { Pathological } \\
\text { fracture }\end{array}$} & \multirow{2}{*}{$\begin{array}{l}\text { Local } \\
\text { recurrence }\end{array}$} \\
\hline & Deltopectoral & $\begin{array}{l}\text { Deltoid } \\
\text { flap }\end{array}$ & & & & & & & & \\
\hline All $(n=37)$ & $10(32 \%)$ & $27(78 \%)$ & $31(84 \%)$ & $35(95 \%)$ & $31(84 \%)$ & $31(84 \%)$ & $14.2(7-21)$ & $5(14 \%)$ & $6(20 \%)$ & $5(14 \%)$ \\
\hline OA $(n=13)$ & $4(31 \%)$ & $9(69 \%)$ & $10(77 \%)$ & $12(92 \%)$ & $10(77 \%)$ & $11(85 \%)$ & $\begin{array}{l}15.4 \\
(10-21)\end{array}$ & $2(15 \%)$ & $2(15 \%)$ & $2(15 \%)$ \\
\hline $\mathrm{APC}(n=10)$ & $5(50 \%)$ & $5(50 \%)$ & $9(90 \%)$ & $9(90 \%)$ & $9(90 \%)$ & $8(80 \%)$ & 12 & 0 & $2(20 \%)$ & 0 \\
\hline $\begin{array}{l}\text { Modular } \\
\text { tumour } \\
\text { prosthesis } \\
(n=14)\end{array}$ & $1(7 \%)$ & $13(93 \%)$ & $12(86 \%)$ & $14(100 \%)$ & $12(86 \%)$ & $12(86 \%)$ & $\begin{array}{l}9.6 \\
\quad(6.5-14)\end{array}$ & $3(21 \%)$ & $2(14 \%)$ & $3(21 \%)$ \\
\hline
\end{tabular}

${ }^{\mathrm{a}}$ Was only used for metastatic disease

humerus using supplemental plate fixation. Compression plate and screw fixation was used for all OA reconstructions.

In the case of the modular tumour prosthesis, we used a Trevira tube surrounding the proximal humeral prosthesis (MUTARS, Implantcast, Buxtehude, Germany) to reattach the principal soft tissues. In allograft use, the surrounding soft tissues were reattached to the appropriate insertion on the allograft.

All were treated with the same postoperative functional regime. For the first four weeks patients were kept in a sling and only pendulum movement was allowed, after which slow progressive exercises were started.

Descriptive statistics were performed for all groups and are presented in Tables 1 and 2. Implant survival was assessed using the Kaplan-Meier method; differences in survival were assessed using the log-rank (Mantel-Cox) test, assuming censored patients (local recurrence or DOD) continued to fail at the same rate as those remaining in the analysis. Preoperative patient characteristics and incidence of complications were assessed using chi-square analysis or Fisher's exact test (e.g. diagnoses, preoperative pathological fractures, abductor compromise, postoperative infection, fracture, instability, non-union, proximal migration, total complications and complications requiring reoperation, prosthesis revision). Correlations between surgical technique, radiological and functional results were assessed using a linear regression analysis. Correlations between proximal migration and functional results were assessed in a subgroup analysis leaving out all patients who had revision surgery, as this was indicated as a significant confounder for functional outcome. Analysis was performed using SPSS Version 17.0 (SPSS Inc., Chicago, IL, USA).

\section{Results}

Postoperative results for all three reconstruction groups are presented in Table 2.

Table 2 Postoperative results

\begin{tabular}{|c|c|c|c|c|c|c|c|c|c|c|}
\hline & $\begin{array}{l}\text { Infection deep/ } \\
\text { superficial }\end{array}$ & $\begin{array}{l}\text { Fracture/ } \\
\text { non-union }\end{array}$ & $\begin{array}{l}\text { Subluxation/ } \\
\text { dislocation }\end{array}$ & Chondrolysis & $\begin{array}{l}\text { Proximal } \\
\text { migration, } \\
n(\%)\end{array}$ & $\begin{array}{l}\text { Surgical } \\
\text { revision, } \\
n(\%)\end{array}$ & $\begin{array}{l}\text { Local } \\
\text { recurrence, } \\
n(\%)\end{array}$ & $\begin{array}{l}\mathrm{DOD} / \\
\mathrm{DOAD}, \\
n(\%)\end{array}$ & $\begin{array}{l}\text { AWOD, } \\
n(\%)\end{array}$ & $\begin{array}{l}\text { MSTS } \\
n(\%)\end{array}$ \\
\hline All & $2 / 1$ & $3 / 2$ & $4 / 2$ & 6 & 20 & 12 & 5 & 10 & 27 & $75.5 \%$ \\
\hline $\begin{array}{l}\mathrm{OA} \\
\quad(n=13)\end{array}$ & $1 / 0$ & $1 / 2$ & $1 / 0$ & $6\left(100 \%{ }^{a}\right)$ & $6(46 \%)$ & $8(61 \%)$ & $3(23 \%)$ & $6(46 \%)$ & $7(54 \%)$ & $77 \%$ \\
\hline $\begin{array}{l}\text { APC } \\
\quad(n=10)\end{array}$ & $1 / 1$ & $2 / 0$ & $3 / 1$ & - & $8(80 \%)$ & $3(30 \%)$ & 0 & $2(20 \%)^{b}$ & $8(80 \%)$ & $72 \%$ \\
\hline $\begin{array}{l}\text { Modular tumour } \\
\text { prosthesis } \\
(n=14)\end{array}$ & $0 / 0$ & 0 & $0 / 1$ & - & $6(42 \%)$ & $1(7 \%)$ & $2(14 \%)$ & $2(14 \%)$ & $12(86 \%)$ & $77 \%$ \\
\hline
\end{tabular}

$n=23$ patients alive with surviving index surgery; OA, $n=6$ [3 patients with no or mild symptoms of chondrolysis (MSTS 74\%), 3 patients with shoulder prostheses inserted (MSTS 79\%)]; APC, $n=7$; EP, $n=10$

$D O D$ died of disease, $D O A D$ died of another disease, $A W O D$ alive without disease

${ }^{\text {a }} 100 \%$ of surviving patients $(6 / 6)$

${ }^{\mathrm{b}}$ One DOD and one DOAD 


\section{Complications}

Non-union, fracture, (sub)luxation and infection were significantly more frequent in patients treated with allograft (OA and APC) $(p=0.03)$. Infection only occurred after revision surgery $(n=3)$; no primary infections were found. Proximal migration was observed in 20 patients with their index surgery still intact (74\%), but was most commonly observed in the APC group $(n=8,80 \%)$.

In five cases (two osteosarcomas and three chondrosarcomas) local recurrence followed after intralesional resection due to the presence of a pathological fracture or insufficient or marginal margins around the neurovascular bundle.

\section{Surgical approach}

The distal deltoid flap approach resulted in a significantly larger percentage of free surgical margins (93 vs $60 \%, p=$ 0.05 ) and an observed higher frequency of an intact and functional abductor mechanism (93 vs 70\%, $p=0.07$ ), when compared to the extended deltopectoral approach. Additionally, it resulted in significantly less proximal migration of the humeral head (62 vs $100 \%, p=0.05$ ). No differences in wide resection margins were observed.

\section{Functional results}

Functional scores for patients treated with OA are provided for all patients left alive in this group $(n=6)$. Three patients were treated for painful chondrolysis using a cemented hemiprosthesis. Of the three patients with their index surgery in situ all presented with chondrolysis. Only one was asymptomatic; the other two preferred not to have revision surgery yet. MSTS scores differed only slightly between these two groups, 74 vs $79 \%$. The presence of proximal migration resulted in significantly lower MSTS scores ( 72 vs $88 \%, p=0.01$ ). Corrected for revision surgery, proximal migration was the only significant negative predictive factor for postoperative MSTS scores $\left(R^{2}=0.4\right.$, $p=0.01)$.

\section{Survival analysis}

Survival analysis using a Kaplan-Meier curve for all three types of reconstruction is presented in Fig. 1. Five-year survival with revision surgery as endpoint for the OA group was significantly lower at $9 \%(n=1)$ when compared to APC $(n=6,60 \%)$ and EPC $(n=8,88 \%)(p=0.001)$. Implant survival with implant removal as endpoint resulted in an implant survival of $100 \%$ in the EP group. This was significantly higher when compared to OA (61\%) and APC $(90 \%)(p=0.002)$. The observed difference between the implant survival for patients with a deltopectoral approach

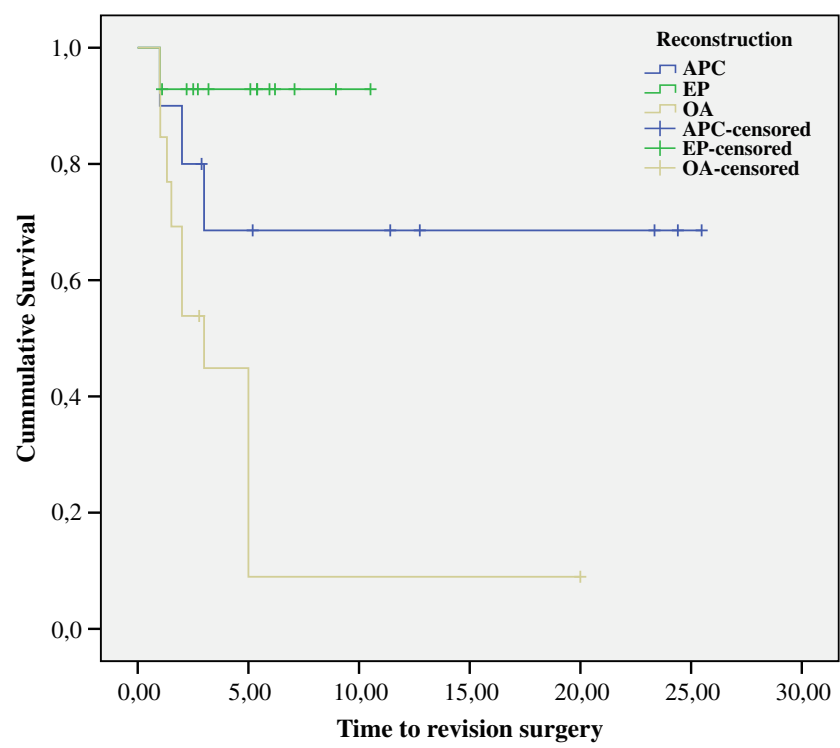

Fig. 1 Kaplan-Meier implant survival curve subgroup analysis for the different types of reconstruction techniques with revision surgery as endpoint

(33\%) compared to a distal deltoid flap approach (61\%) was also significant ( $p=0.03)$ (Fig. 2).

\section{Discussion}

Proximal humeral reconstruction has anecdotally been reported to have serious postoperative functional deficit and high complication rates. The use of allograft is frequently promoted for restoration of bone stock and anatomical reconstruction of soft tissues. Some studies have stated that this anatomical repair resulted in better postop-

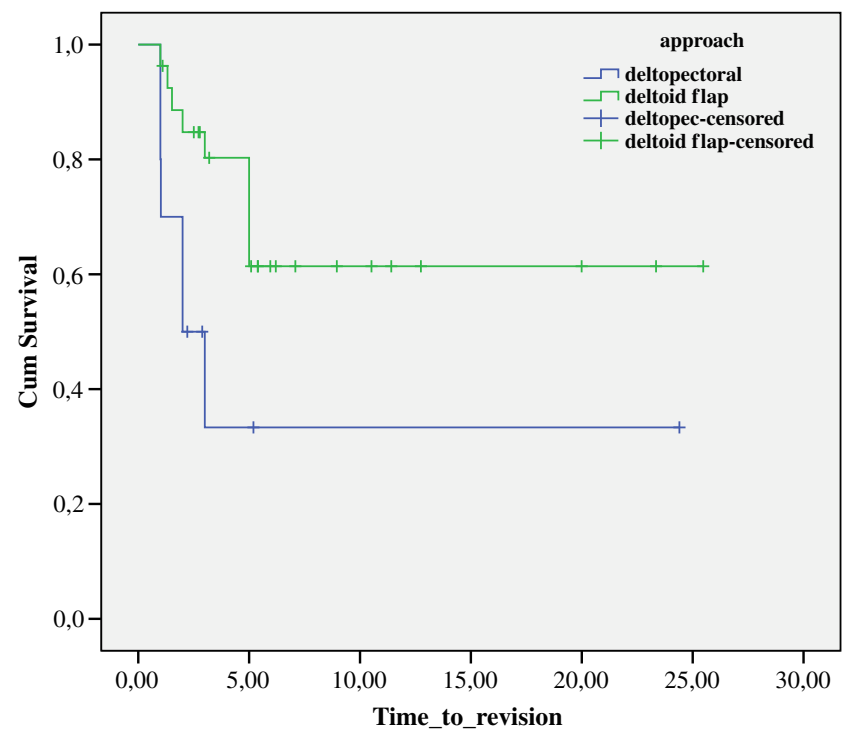

Fig. 2 Kaplan-Meier implant survival subgroup analysis for surgical approach with revision surgery as endpoint 
erative function and glenohumeral stability [4, 8]. In contrast, chondrolysis, allograft fracture and non-union have also been introduced as reasons not to consider OA for standalone proximal humeral reconstruction [14-16]. The additional use of a cemented prosthesis into the allograft, preferably bridging the allograft and fixed into the healthy autologous humeral bone, should prevent chondrolysis and allograft fracture but are still susceptible to non-union, osteolysis and loosening [2, 4, 7]. Modular mega-prostheses for proximal humeral reconstruction presented a solution for complications inherent to biological repair, but were presumed prone to (sub)luxation and offered less healthy bone to work with when revision surgery was warranted [4]

We postulated that use of an EP alone would diminish surgical complications and increase implant survival. In addition, we assumed that the use of a deltoid flap approach would achieve better abductor mechanism and rotator cuff preservation leading to improved functional results and implant survival.

Our series of proximal humeral reconstruction has underlined the high complication risk for OA use alone. The use of allograft combined with a primary cemented shoulder prosthesis did significantly improve implant survival, but was still subject to a higher number of surgical complications in need of surgical revision, when compared to EP use alone. Additionally, it showed that functional results and MSTS scores for the use of EP were not inferior to that of APC reconstructions. Mid- and long-term eventfree implant survival was significantly better for EP use alone. When compared to findings in recently presented studies, we found comparable functional results and implant survival statistics for all three groups (Table 3) [1-7]. However. functional comparison remains very much dependent on tumour localisation, joint and soft tissue involvement, use of postoperative radiotherapy and was reported using several outcome scores. It was therefore not included in Table 3 [2, 4, 6, 7, 17].

It must be noted that time set and local preference for one or the other technique should be regarded as major confounders of postoperative results and implant survival.

Assessment of the two different surgical approaches (extended deltopectoral and distal deltoid flap) presented an observational difference in implant survival and significant difference in abductor mechanism preservation, proximal migration and surgical resection margins all in favour of the deltoid flap approach. The distal deltoid flap approach presented us with a safe and reliable surgical exposure of the proximal humerus providing for wide resection of bony and soft tissue tumour components, without compromise of the deltoid muscle, axillary nerve and rotator cuff muscles. We believe this may explain the significant difference in the incidence of proximal migration.

\section{Study limitations}

Although our results did support our study hypothesis, conclusions drawn must be read in light of our study limitations. The primary limitation must be its retrospective character and inevitable small numbers. Additionally the multiform patient characteristics and surgical techniques account for the limited statistical importance of this paper. Still the clear and significant differences in implant survival and complication rates put forward a slight preference towards prosthetic use alone in proximal humeral reconstruction. Although no patients were lost to follow-up, only 27 patients were alive without disease during final follow-up. Of these, only 23 patients still had their index surgery in place. This must have introduced a serious confounder due to selection bias as non-survivors were assumed to have continued to fail at the same rate as those remaining in the analysis.

Additionally, the superior results for the deltoid flap approach when compared to the deltopectoral approach may have been subject to some indication bias as the deltoid flap approach was probably not used when tumour boundaries included the deltoid muscle itself. Finally, the time frame of the study does introduce bias due to surgical experience, new surgical techniques and materials, but this is inevitable when reporting on the treatment of this relatively rare tumour location.

\section{Complications}

Although infection is regarded as a major complication of allograft and prosthetic reconstruction after limb-salvage surgery, this was only rarely seen in our series. All three deep infections occurred after revision surgery for other reasons and this was coherent with recent literature $[4,7]$.

Table 3 Overview of recent literature for the three types of reconstruction $[2,4,6,7,14,16]$

\begin{tabular}{llllll}
\hline Reconstruction & $n$ & Mean follow-up (months) & Complication rate (\%) & Reoperation rate (\%) & Implant survival (without revision) (\%) \\
\hline Allograft & 52 & 72 & 49 & 36 & 62 \\
Composite & 52 & 76 & 40 & 21 & 88 \\
EPR & 149 & 43 & 11 & 7 & 91 \\
\hline
\end{tabular}


In only one of these patients could the index reconstruction be preserved; the other two were revised with the use of an EP.

Glenohumeral instability and proximal migration were assumed to be less frequent in allograft use as musculotendinous reconstruction would be superior when compared to EP use alone [4, 7]. Our results do not support this conclusion as instability and proximal migration were equally divided in both APC and EP groups. The use of an periprosthetic Trevira tube to securely reattach the deltoid, pectoral and rotator cuff muscles around the proximal humeral prosthesis may have improved our results [18].

\section{Authors' conclusions}

The retrospective character and small numbers of all studies including our own limit the possibility for specific subgroup analysis to assess the influence of patient characteristics, surgical technique and implant choice on the reconstruction and tumour-related complications, functional outcome and reconstruction survival. Specific factors improving functional results and decreasing the need for surgical revision are undoubtedly imminent to provide surgeons and patients alike with the appropriate choice of proximal humerus reconstruction. Our results support the use of EPR after tumour resection of the proximal humerus over APC and OA reconstructions, because implant survival is superior and complication rates necessitating revision surgery much lower, without negatively influencing the postoperative functional results. Additionally, we would advocate a surgical technique that poses the least danger of abductor mechanism compromise, offers sufficient surgical exposure and presents safe possibilities for wide surgical margins [1]. The use of the distal deltoid flap approach is therefore advocated.

Open Access This article is distributed under the terms of the Creative Commons Attribution Noncommercial License which permits any noncommercial use, distribution, and reproduction in any medium, provided the original author(s) and source are credited.

\section{References}

1. Gupta GR, Yasko AW, Lewis VO et al (2009) Risk of local recurrence after deltoid-sparing resection for osteosarcoma of the proximal humerus. Cancer 115:3767-3773
2. Cannon CP, Paraliticci GU, Lin PP, Lewis VO, Yasko AW (2009) Functional outcome following endoprosthetic reconstruction of the proximal humerus. J Shoulder Elbow Surg 18:705-710

3. Gosheger G, Hardes J, Ahrens H, Gebert C, Winkelmann W (2005) Endoprosthetic replacement of the humerus combined with trapezius and latissimus dorsi transfer: a report of three patients. Arch Orthop Trauma Surg 125:62-65

4. Abdeen A, Hoang BH, Athanasian EA, Morris CD, Boland PJ, Healey JH (2009) Allograft-prosthesis composite reconstruction of the proximal part of the humerus: functional outcome and survivorship. J Bone Joint Surg Am 91:2406-2415

5. DeGroot H, Donati D, Di Liddo M, Gozzi E, Mercuri M (2004) The use of cement in osteoarticular allografts for proximal humeral bone tumors. Clin Orthop Relat Res 427:190-197

6. Rödl RW, Gosheger G, Gebert C, Lindner N, Ozaki T, Winkelmann W (2002) Reconstruction of the proximal humerus after wide resection of tumours. J Bone Joint Surg Br 84:10041008

7. Potter BK, Adams SC, Pitcher JD Jr, Malinin TI, Temple HT (2009) Proximal humerus reconstructions for tumors. Clin Orthop Relat Res 467:1035-1041

8. Black AW, Szabo RM, Titelman RM (2007) Treatment of malignant tumors of the proximal humerus with allograftprosthesis composite reconstruction. J Shoulder Elbow Surg 16:525-533

9. Mayilvahanan N, Paraskumar M, Sivaseelam A, Natarajan S (2006) Custom mega-prosthetic replacement for proximal humeral tumours. Int Orthop 30:158-162

10. Enneking WF, Dunham W, Gebhardt MC, Malawar M, Pritchard DJ (1993) A system for the functional evaluation of reconstructive procedures after surgical treatment of tumors of the musculoskeletal system. Clin Orthop Relat Res 286:241-246

11. Rispoli DM, Athwal GS, Sperling JW, Cofield RH (2009) The anatomy of the deltoid insertion. J Shoulder Elbow Surg 18:386-390

12. Glanzmann MC, Goldhahn J, Flury M, Schwyzer HK, Simmen BR (2010) Deltoid flap reconstruction for massive rotator cuff tears: mid- and long-term functional and structural results. J Shoulder Elbow Surg 19:439-445

13. Martini M (1976) The sub-deltoid approach to the metaphyseal region of the humerus. J Bone Joint Surg Am 58:377-379

14. Kumar VP, Satku K (1992) Osteoarticular allografts for reconstruction in the proximal part of the humerus after excision of a musculoskeletal tumor. J Bone Joint Surg Am 74:152

15. Getty PJ, Peabody TD (1999) Complications and functional outcomes of reconstruction with an osteoarticular allograft after intra-articular resection of the proximal aspect of the humerus. J Bone Joint Surg Am 81:1138-1146

16. Gebhardt MC, Roth YF, Mankin HJ (1990) Osteoarticular allografts for reconstruction in the proximal part of the humerus after excision of a musculoskeletal tumor. J Bone Joint Surg Am 72:334-345

17. Raiss P, Kinkel S, Sauter U, Bruckner T, Lehner B (2010) Replacement of the proximal humerus with MUTARS tumor endoprostheses. Eur J Surg Oncol 36:371-377

18. Marulanda GA, Henderson E, Cheong D, Letson GD (2010) Proximal and total humerus reconstruction with the use of an aortograft mesh. Clin Orthop Relat Res 468:2896-2903 